\title{
Alianza estratégica entre Venezuela y Nicaragua para la construcción de una refinería petrolera
}

Dani José Villalobos Soto ${ }^{1}$

\section{Resumen}

El comercio internacional tiene una naturaleza cambiante y dinámica por lo cual ha requerido de constante revisión acerca de las estrategias para mantener y expandir los nexos comerciales, que siempre se dirigen a las ventajas absoluta, comparativa y competitiva. A través del tiempo se ha demostrado que la esencia misma del comercio se encuentra en la combinación de dichos factores y las acciones que los países toman para perfeccionar y especializarse en la producción de bienes y servicios. En la premisa que cada país se concentra en dedicar mayores esfuerzos a producir los bienes que por distintas razones ya sean geográficas, climáticas o tecnológicas, se establece la descripción de la Alianza Estratégica entre Venezuela y Nicaragua para la construcción de una refinería petrolera considerando las potencialidades de Venezuela como principal productor y proveedor de petróleo y derivados especialmente combustibles de la Cuenca del Mar Caribe; pero, con significativas debilidades en la producción agropecuaria; mientras que Nicaragua es un relevante productor agrícola y ganadero en Centroamérica, aunque con carencia de combustibles y vías de comunicación terrestre que limitan el comercio. El abordaje metodológico es descriptivo desde un nivel explicativo; por ello el autor propone en el contexto de PETROALBA profundizar en los principios de equidad y complementariedad. Se formuló como conclusión principal que las debilidades y potencialidades se convierten en factores primordiales para incentivar el comercio y alcanzar mercados estables y seguros.

Palabras Clave: Refinería petrolera; comercio internacional; principios de equidad; complementariedad.

\section{Abstract}

International trade has a changing and dynamic nature which has required a constant review of strategies to maintain and expand trade links, which are always directed at absolute, comparative and competitive advantages. Over time, it has been shown that in the main of trade, lies the combination of these factors and the actions that countries take to perfect and specialize in the production of goods and services. On the premise that each country concentrates on making greater efforts to produce goods that for different reasons, whether geographic, climatic or technological, establishes the description of the Strategic Alliance between Venezuela and Nicaragua for the construction of an oil refinery considering the potentialities of Venezuela as the

1 Profesor Investigador de la Universidad Simón Bolívar. Correo: dvillalobos@usb.ve

Recibido: 25/04/2017 Aprobado: 16/08/2017

Villalobos, D., J. (2017). Alianza estratégica entre Venezuela y Nicaragua para la construcción de una refinería petrolera. Ciencia e 
main producer and supplier of oil and especially fuel derivatives in the Caribbean Sea Basin; But with significant weaknesses in agricultural production; While Nicaragua is a major agricultural and livestock producer in Central America, although lacking fuels and terrestrial communication channels that limit trade, the methodological approach is descriptive from an explanatory level; For this reason, the author proposes in the context of PETROALBA, to look deeply into the principles of equity and complementarity. The main conclusion was that weaknesses and potentialities become key factors in stimulating trade and achieving stable and secure markets.

Keywords: Oil refinery; International Trade; Principles of equity; Complementarity.

\section{Introducción}

La dinámica del comercio, presenta un comportamiento con una vinculación estrecha a los principios que rige la economía como disciplina; en todo intercambio comercial se evidencia las potencialidades y debilidades que presentan las empresas, o países cuando se refiere al comercio internacional, precisamente la esencia del mismo se concentra en adquirir productos y servicios que no se producen en un lugar determinado para satisfacer toda la demanda interna o comprar los bienes y servicios que no se producen.

Por ello, los enunciados de la ventaja comparativa y competitiva que explicó Adam Smith en el siglo XVII no han perdido vigencia e implica uno de los factores fundamentales para explicar la integración regional como el mecanismo apropiado para estimular el comercio justo, equitativo en el entorno de la cooperación y del regionalismo, cabe destacar que los acuerdos de integración se deciden y se celebran en un contexto mucho más complejo que la cooperación porque implica la afinidad de intereses que tienen los países ya sea la necesidad del intercambio comercial, de establecer vías de comunicación terrestres, prevenir los actos ilícitos como el contrabando en todo caso es una tendencia constante que la integración es una expresión de intereses comunes.

América Latina y el Mar Caribe, con las Antillas Mayores y Menores constituyen una de las regiones más estratégicas y con una posición privilegiada que favorece el comercio internacional, las comunicaciones y el transporte de mercancías. En dicho contexto Venezuela se destaca como el país con fachada frontal hacia el Gran Caribe (Cuba, Puerto Rico, y República Dominicana ), así como al Caribe Insular: Bahamas, Granada, Barbados, Dominica, Santa Lucía, Trinidad y Tobago, San Vicente y las Granadinas, Antigua y Barbuda y San Cristóbal y Nieves, también destaca el hecho que Venezuela es el país caribeño con la mayor producción petrolera y por lo tanto, principal proveedor de la región. 
Se considera que Venezuela, es un país cuya principal potencialidad económica lo constituyen el petróleo y derivados, con una posición geográfica predominante y privilegiada en el Mar Caribe, pero presenta debilidades en la producción agrícola y ganadera, cuya producción interna no satisface toda la demanda de recursos mientras que la República de Nicaragua dispone de manera satisfactoria para cubrir dicha demanda de Venezuela en este renglón.

Por consiguiente, uno de los factores que ha incidido desfavorablemente en el estímulo a la industrialización y desarrollo en Centroamérica es la escasez de combustibles, y vías de comunicación terrestres, el siguiente artículo de investigación se inicia con la premisa de: Investigar la Alianza Estratégica entre Venezuela y Nicaragua para la construcción de una refinería petrolera por lo cual significa: la creación de nuevas fuentes de empleo en la costa del Océano Pacífico de Nicaragua con la asesoría de expertos petroleros venezolanos y así facilita la distribución de combustibles en Nicaragua.

A partir de la anterior explicación, surgen las siguientes interrogantes que constituyen el objeto de estudio de la siguiente investigación: ¿El hecho de establecer alianzas estratégicas es un contrasentido con la ventaja comparativa y competitiva? ¿Aliarse anula las posibilidades de competir? las propuestas, los mecanismos de intercambio comercial, e incluso las posibilidades de generación de empleo en el contexto de Efecto Multiplicador atañen a la gerencia.

\section{Desarrollo}

\section{Justificación de la investigación}

La cooperación económica, responde a una naturaleza propia que consiste en los principios de solidaridad, equidad y complementariedad que se alinea en concordancia con PETROALBA, una iniciativa innovadora que despierta el interés del investigador por conocer sus alcances y limitaciones y sobre todo aportar en su estudio al fortalecimiento de la región latinoamericana y del Caribe con el objetivo de afianzar las fortalezas y corregir las debilidades.

Por el hecho que es un tema de reciente investigación, se requirió identificar a partir de la revisión exhaustiva de distintas fuentes de información, las variables tales como: ventaja comparativa y ventaja competitiva, el efecto multiplicador y alianza estratégica entre Venezuela y Nicaragua. En la dinámica misma de la investigación, surgen un conjunto de razones que le conceden relevancia de tal manera, que alcanzan trascendencia para que otras personas continúen el tema.

La investigación se considera relevante, ya que se concentra en demostrar la concordancia, la factibilidad y beneficios en términos del Efecto Multiplicador de la 
construcción de una refinería en la costa del Océano Pacífico nicaragüense cuyo propósito esencial consiste en generar nuevas fuentes de empleo y mayor capacitación en el área petrolera con la asesoría de expertos petroleros venezolanos.

\section{Alianzas estratégicas, en el comercio internacional como factor de la gerencia}

Si se parte de la premisa, que en toda actividad inherente al comercio internacional, se apoya y complementa especialmente en acuerdos de la integración de alcance extenso o reducido, pero cuya esencia implica la cooperación lo cual conduce a la coordinación de actividades para lograr metas predeterminadas ya sean etapas de la integración; zonas aduaneras, preferencias arancelarias, libre comercio, o la complementación intrínseca de la ventaja comparativa y competitiva entre otros en un sentido general, el núcleo de la investigación encuentra en demostrar la vinculación entre:

- Ventaja absoluta.

- Ventaja comparativa.

- Ventaja competitiva de las naciones

- Alianza estratégica.

- Teorías del desarrollo.

- PETROALBA.

- Comercio.

Para establecer vínculos en los conceptos mencionados, el comercio internacional es una constante interacción en la cual intervienen la cooperación, negociación, acuerdos, ventajas absolutas, competitivas y ventajas comparativas; además, que prevalece la búsqueda de alianzas más que de competencias para alcanzar metas en común, en este sentido el comercio propicia el equilibrio entre bienes y servicios que se demandan y se producen; los esquemas de integración han el hecho de cambiar la percepción del comercio en términos de cooperación y no en términos de estricta competencia razón por la cual se destaca la definición que expone Hindle (2008) acerca de Alianza Estratégica:

Es un tipo de relación entre dos o más organizaciones que está entre un acuerdo de tercerización en el que las empresas participantes se encuentran en pie de igualdad y una verdadera adquisición. Incluye la concesión de franquicias, licencias y la unión transitoria de empresas (p. 205).

En ese sentido, el autor considera pertinente hacer una extrapolación y comparar el planteamiento con los vínculos entre Estados en esquemas integracionistas, específicamente PETROALBA y la relación comercial entre Venezuela y Nicaragua; cuando en el mismo tratado se expresa "en Pie de Igualdad" se refiere a las potencialidades que 
tiene cada país para estimular el intercambio comercial y como cada uno ha desarrollado la especialización en un sector determinado; Venezuela en petróleo y derivados y Nicaragua en ganadería y agroindustria, continua Hindle en la explicación:

Un acuerdo cooperativo entre dos o más compañías en el que se desarrolla una estrategia común en conjunto y todas las partes adoptan una actitud de beneficio mutuo. La relación es recíproca, y cada socio se prepara para compartir fortalezas específicas con el otro, y así dar poder a la empresa. Existe un uso colectivo de recursos, inversión y riesgo para obtener una ganancia mutua (Hindle, 2008).

Las alianzas estratégicas, constituyen acuerdos, en los cuales las partes involucradas han identificado y determinado que presentan intereses en común, y les conviene más cooperar entre sí que competir irracionalmente, lo cual a su vez significa uno de los principios fundamentales de la integración, que se apoya en el consenso más que en la competencia y aun así en términos equitativos las alianzas son una unión de intereses y metas comunes que no sólo se aplica a las empresas, también a los Estados como entes públicos garantes del bienestar ciudadano y gerente de los patrimonios nacionales.

Además, las alianzas desarrollan un conjunto de funciones que potencian los objetivos de las instituciones ya que consisten en una red de interacciones en la sociedad que unen instituciones de diversa naturaleza, propicia la cooperación, se divulgan innovaciones tecnológicas y nuevos productos, amplían distintos enfoques, el hecho de aliarse constituye un mecanismo óptimo para estimular el comercio regional e internacional, le imprime dinamismo y necesariamente actualización y competitividad factores que contribuyen a mantener la naturaleza propia del comercio.

En otro orden de ideas, se logran objetivos de distinta índole, en la extensión de dicha interacción surgen procedimientos en los cuales las empresas representativas tiene la posibilidad de captar nuevos mercados en otros países participantes, así se demuestra que la alianza estratégica no es un mecanismos exclusivos de las empresas privadas; también aplica a las instituciones públicas y la esencia misma del Estado como principal negociador y promotor del comercio.

\section{La ventaja absoluta como premisa inicial}

Como una breve referencia desde el siglo XV, cuando los mercados europeos comenzaron a ampliar a través de la conquista de América, la posibilidad establecer relaciones comerciales, cuyas operaciones se cancelarán en monedas y especias se concentró la atención del continente en mejora las condiciones de producción de los bienes que por distintas razones se producían en mayor cantidad y calidad, se destaca la definición que aporta Parkin (2006): 


\section{CIENCIAS SOCIALES}

Se tiene ventaja absoluta cuando se puede producir más bienes a partir de una cantidad específica de recursos que cualquier otro individuo. La ventaja absoluta es el resultado de las diferencias entre la productividad. Una persona cuenta con mejor tecnología, más capital o mejores habilidades que otras, tiene una ventaja absoluta con respecto a esta última (p. 43).

La cita anterior contribuye a realizar una comparación, entre las facilidades naturales que tiene cada país para producir determinados bienes; en esencia Venezuela es un país petrolero, en cuyos suelos por millones de años, como resultado de la materia orgánica se ha formado petróleo de distintas categorías, ya sea ligero o extrapesado, así como yacimientos de gas natural que se ha transformado en un combustible muy preciado, dicha premisa conduce a inferir que el espacio para las actividades agrícolas y pecuarias existen pero no son extensivas, lo cual coloca a Venezuela como el principal productor petrolero y proveedor de combustibles y derivados de la cuenca del Mar Caribe y Centroamérica, a excepción de Trinidad y Tobago, así Venezuela tiene ventaja absoluta en la producción petrolera.

En el caso de Nicaragua, la potencialidad se perfila esencialmente agropecuaria, es el país centroamericano junto con Costa Rica y Panamá que sufrió con menos rigor los efectos de las guerras civiles en las décadas de 1970 y 1980, lo cual le permitió por muchos años mantener determinada estabilidad económica, así como la extensión de las actividades ganaderas y agroindustriales, potencialidades que en la actualidad se convierten en la estrategia más óptima para participar en la dinámica del comercio regional, especialmente cuando Nicaragua presenta debilidades en la distribución de petróleo, derivados y combustibles, y no es en realidad un país productor petrolero, se destaca la explicación de Calvo (2016):

La división del trabajo favorece la especialización, según las aptitudes llevando a un incremento de la producción y a un mayor volumen de intercambio. Para Adam Smith estaba muy claro que un país ganaba con el comercio; cada país podrá producir y comerciar el bien en cuya producción tenga una ventaja en términos de coste (p. 21).

La esencia de la ventaja absoluta se encuentra, en mejorar la eficiencia que cada país tiene en la producción de determinados, como la naturaleza del comercio es dinámica, de ella provienen las ventajas comparativas y competitivas que se refieren principalmente a las estrategias y mecanismos que se aplican para producir y mantener los bienes en el mercado y mantenerse en equilibrio con la producción de otros países o empresas. 


\section{Las ventajas comparativas y ventajas competitivas en el acuerdo PETROALBA de la República Bolivariana de Venezuela y de Nicaragua}

Desde el siglo XVII, a través de los estudios de economistas como Adam Smith y David Ricardo quienes explicaron que las causas del comercio internacional se encuentran en las facilidades naturales que tiene cada país para producir determinados tipo de bienes, dicha categoría de especialización, es lo que permite que unos países importen los bienes que no producen o no producen en cantidades suficientes para satisfacer la demanda interna y exporten los bienes que producen con calidad de excedentes, además así se presenta la opción de mejorar y perfeccionar progresivamente dicha producción.

En términos prácticos, no es una condición indispensable que un país debe presentar la inexistencia en la producción de un bien, con la categoría que la producción es insuficiente es apropiado para los mecanismos del comercio internacional, en el sentido que la dinámica del mismo radica, en el requerimiento que tienen unos países de comprar y otros países de vender destacando la características naturales unos tendrán más facilidades para la producción agrícola, mientras que otros tienen una dotación natural de hidrocarburos o minerales como es el caso de Venezuela, Churión (2001) lo explica en los siguientes términos: "Ventajas Comparativas Absolutas es cuando un país se dedica a producir aquel bien o servicio en el cual tiene costos más bajos al compararse con el de otros países" (p. 93).

Si se aplica el anterior enunciado, al caso del presente estudio se establece que la ventaja comparativa de Venezuela, es la producción de hidrocarburos combustibles y minería, lo cual ha generado la continua renta petrolera y contribuye al desarrollo general del país, en el sector minerales, que en el caso de Nicaragua presenta la debilidad de Centroamérica y el Caribe que es la escasez de producción en hidrocarburos y combustibles en una amplia gama de producción agrícola por lo cual el vínculo comercial consiste en el intercambio de combustibles por bienes agrícolas aunque Venezuela los produce no constituye la mayor fortaleza de la economía nacional; de la ventaja comparativa deriva la Ventaja Competitiva que lo define Churión (2001) en los siguientes términos:

Las ventajas competitivas resultan principalmente de una rápida innovación y mejoramiento continuo; no es permanente ésta puede perderse si la empresa se estanca tecnológicamente...En consecuencia el desarrollo tecnológico de un país le permite desarrollar ventajas competitivas que pueden neutralizar las ventajas absolutas de otro (p. 94).

En la actualidad, el avance de la tecnología y globalización ha contribuido significativamente a modificar las debilidades que un país presentase en el sistema económico y sustituirlas por nuevas ventajas, por ejemplo, la ágil industrialización en diez años 
del Sudeste Asiático, convirtió a países subordinados al colonialismo anglo francés cuyas economías eran esencialmente agrícolas a economías que se destacan por la producción manufacturera, textil y tecnológica.

En el contexto de la integración regional y la cooperación, se percibe como una gran contradicción la noción de competencia y competividad con el intercambio comercial en una región como América Latina pacifista y proclive al consenso y la cooperación; sin embargo, es precisamente en la necesidad de complementar la producción que escasea en algunos países de la región que se concentra la esencia del Acuerdo ALBA en el intercambio de bienes y servicios para satisfacer la demanda interna; Venezuela ha desarrollado por décadas rubros agrícolas como el café y el cacao pero no es la principal potencialidad del país, cuya naturaleza es esencialmente petrolera y minera, al contrario de Nicaragua con amplio desarrollo agrícola, nula producción petrolera y minera.

\section{La ventaja competitiva de las naciones en la concepción de Michael Porter}

En la naturaleza propia del comercio internacional tan dinámica, y demandante de innovaciones el autor Michel Porter, introdujo importantes aportes desde 1991 cuando formuló una explicación distinta al enunciado clásico de Adam Smith y David Ricardo, en el sentido que describe como la innovación, y el dominio de la tecnología son condiciones significativas de la participación de los países en el comercio y en el volumen de importaciones y exportaciones, por lo cual la explicación es mucho más profundo que el hecho de comparar producciones y se refleja en la siguiente cita por León referente a por Porter (1991) :

La competencia es dinámica y evolutiva: Una nueva teoría debe reflejar un rico concepto de la competencia que comprenda los mercados segmentados, los productos diferenciados, las diferencias en las tecnologías y en las economías de escala. La calidad, las características, la innovación en los nuevos productos son determinantes en los sectores y segmentos avanzados...La competencia es un paisaje sometido a constante cambio. En el que continuamente afloran nuevos productos, nuevas formas de comercializarlos, nuevos procesos de fabricación y segmentos de mercados completamente nuevos (p. 46).

Desde esta perspectiva, la competitividad para que sea perdurable en el tiempo tanto para los países, como para las empresas requiere estrategia de innovación, lo cual conlleva al estudio permanente de los mercados para conocer todas las expectativas necesidades y requerimientos que se presenten; en este sentido, la explotación petrolera ofrece una amplia gama de posibilidades a través de los productos derivados, combustibles, plásticos y siliconas. De allí la propuesta de una refinería que no solamente genere producción sino también fuentes de empleo en zona de Nicaragua que 
ha sido tradicionalmente agrícola, así por parte de Venezuela se cumple la premisa de la innovación y el estudio constante acerca de la creación de nuevos derivados del petróleo.

Además, en la perspectiva de Nicaragua, existen factores que favorecen la competitividad tales como fue el país menos afectado por la guerra civil de la década de 1980, presenta la producción ganadera y agrícola más importante de Centroamérica con una posición geográfica privilegiada cercana al Golfo de México, Estados Unidos y el Canal de Panamá, dispone de costas en el Océano Pacifico y Mar Caribe, lo cual es una vía de conexión directa con el Sudeste Asiático y Sudamérica, respectivamente y con Norteamérica y los países caribeños; el principal potencial nicaragüense lo constituye la producción agropecuaria, la insuficiente red de vías de comunicación terrestres y la falta de combustibles ha retrasado la expansión de dicha industria.

Con la construcción de la refinería en la costa norte del pacífico de Nicaragua, se fortalecen la oportunidades para pequeños y medianos productores, así como empresas más grandes para colocar los productos en calidad de exportación a través de la vías de comunicación que se construyan, especialmente una vía que conecte ambas costas a través de los departamentos de la Región Autónoma de la Costa Caribe Norte RACCN, Jinotega, Estelí, León y Nueva Segovia; de esta forma ambas naciones tiene la oportunidad de competir en términos distintos, equitativos e innovadores.

\section{La integración regional y la cooperación como factor primordial para lograr el consenso}

América Latina y el Caribe, han experimentado cambios en sus formas de establecer relaciones comerciales. La región ha dejado las estrategias de desarrollo orientadas hacia adentro con un mercado interno que muestra significativos signos de intervención y un Estado protagónico, y ha asumido una estrategia de desarrollo orientada hacia los mercados externos, por lo tanto, la asignación de recursos es determinada básicamente por el mercado, y donde el agente principal de desarrollo es la empresa privada.

Es sumamente importante destacar que, el ALBA se constituye como una nueva mecánica de integración de cooperación cuyo propósito es la de aprovechar las ventajas cooperativas entre las naciones que conforman el bloque y de esta manera dar respuesta efectiva a las asimetrías que caracterizan a cada uno de estos países que conforman dicho esquema, para efectos de mayor compresión se incluye la definición de integración regional por Rodríguez (2010): la integración regional constituye un esfuerzo o movimiento convergente realizado por parte de los Estados, tendente a la obtención de objetivos comunes, mediante la armonización o unificación no sólo de políticas económicas y financieras, de regímenes monetarios, sino también de políticas socio-culturales y legislativas. 
De acuerdo con la cita anterior la integración, se concibe como un proceso progresivo, dinámico, concordante con los intereses nacionales y colectivos de los estados cuya principal esencia, se encuentra en la cooperación como acto de voluntad y la disposición a innovar y a crear espacios para el consenso, pero dicha descripción no se restringe a enunciados teóricos sino en el hecho de aplicarlo en la realidad; se evidencia en la eficiencia y cumplimiento de expectativas que se tiene, en ocasiones la integración no es un acto meramente comercial o económico, por el contrario se inicia en la convicción, cooperación y disposición al diálogo.

La acción que demuestra el verdadero éxito de un proceso integracionista, se encuentra en los resultados que se comprueban en las comunidades, estructuras económicas, y en el comercio. Un acuerdo de integración consiste en un contrato en términos jurídicos, pero en términos pragmáticos constituye un conjunto de actividades que contribuyen a mejorar las condiciones de vida de la población e incluso a concientizar dichos procesos como parte dinámica de la existencia cotidiana. La integración es eficiente cuando la rentabilidad del comercio, se incrementa al igual que las opciones de empleo, producción, ingresos también aumentan y así se generan mayores recursos monetarios que directa o indirectamente mejoran todas las condiciones en general.

En una definición más simple y en el marco del Derecho Internacional Público se establece que la cooperación internacional es un objetivo de los Estados para buscarle solución, mediante una acción colaboracionista, a los más ingentes problemas de la humanidad (Rodríguez, 2010). Además, la cooperación se evidencia, en las normas de derecho cuando la misma se codifican a través de Tratados, Acuerdos y Convenios que expresan la voluntad y disposición de los Estados en determinar acuerdos en una materia específica. Según esta definición y como es mencionado anteriormente PETROALBA, como Acuerdo de Cooperación Energética promueve el desarrollo económico entre los países que lo conforman, y no sólo eso sino también de la Comunidad Internacional y el Progreso del Derecho Internacional; con la naturaleza de dicho acuerdo se demuestra que es posible la convergencia entre cooperación, justicia, equidad e intereses nacionales de una forma equilibrada con la tendencia a mejorar la calidad de vida y entorno en general de los países participantes.

\section{Teorías del desarrollo}

Las perspectivas de la teoría del desarrollo permiten no solo aclarar conceptos y analizarlos, sino también identificar recomendaciones relacionadas como políticas sociales. Se destaca la siguiente definición de Desarrollo Económico por Pérez (2004): "Son los cambios en el seno de las sociedades pobres para alcanzar el progreso material... Es un proceso en el que la expansión de la producción viene acompañada de transformaciones en las estructuras económicas y sociales" (p. 7). 
Según la definición anterior existen aspectos en los que se interna lo siguiente: en el sentido económico se comprende que un país si existen oportunidades de empleo, satisfacción de las necesidades básicas y una tasa positiva de distribución y redistribución de las riquezas. En el aspecto político se puede notar que los sistemas de gobierno tienen legitimidad legal para proporcionar beneficios sociales a sus pobladores, sin embargo, la magnitud y la dimensión del término desarrollo presenta varias facetas, como lo explica Ray (2001):

El desarrollo es la eliminación de la pobreza y de la desnutrición: es un aumento de la esperanza de vida; es el acceso a la red de saneamiento, al agua potable y a los servicios sanitarios, es la reducción de la mortalidad infantil, es un mayor acceso al saber, a la educación y a la posibilidad de aprender a leer y escribir (p. 6).

El desarrollo no debe proyectarse como una meta exclusiva, cuyo fin consista solamente en llegar, por el contrario para que un país tenga desarrollo, se refiere a un conjunto de factores que se relacionan entre sí entre medidas económicas y políticas acertadas continuistas y coherentes; implica un proceso progresivo que incluso se extiende por años o siglos, que requiere en ocasiones determinadas políticas expansionistas ya sea en términos territoriales, geográficos y el más relevante de todos comerciales. Los países desarrollados son los que mantienen mayores vínculos comerciales con el resto del mundo.

Dichos factores son amplios y complejos, ya que el desarrollo incluye la cultura, principios, la percepción que tienen los ciudadanos tanto de sí mismos como del país, los recursos naturales, como se aprovecha la posición geográfica, y por último cuales son las medidas que aplican los gobernantes para propiciar el desarrollo, y en ello tiene una relevancia vital el comercio; cuales son los modelos de comercio que se asuman para lograr el desarrollo; si se promociona un bien en especial, si se promocionan el país como un lugar idóneo para realizar inversiones, si se hace concesiones a empresa transnacionales para explotar recurso; en todo caso, el desarrollo comienza por una aptitud individual, colectiva y nacional para después crear las condiciones apropiadas.

\section{Conclusiones}

De acuerdo a las explicaciones expuestas, es necesario considerar convenientemente formular un espacio de conclusiones acerca de la Alianza Estratégica entre Venezuela y Nicaragua, ya que el comercio internacional se caracteriza por ser altamente dinámico y demandante de innovaciones, y nuevos sistemas de operatividad y logística.

En la alianza estratégica entre Venezuela y Nicaragua, se evidencia como las debilidades y potencialidades de los países se convierten en relevantes factores que contribuyen a incentivar el comercio y alcanzar mercados estables y seguros; la principal potencialidad de Venezuela lo constituye el petróleo y derivados , así es 
el principal proveedor de la cuenca del Mar Caribe y Centroamérica, pero presenta debilidades en la agricultura y ganadería, situación que favorece a Nicaragua como principal proveedor de Venezuela de dichos rubros y a su vez no produce petróleo, y presenta serias carencias en la distribución de combustibles.

Los acuerdos integracionistas, no se reducen exclusivamente al acuerdo jurídico, o más concretamente a la firma del contrato que se expresa en el Tratado Internacional; existe toda una fase previa que implica el dominio de la información, investigación, la determinación de oportunidades de negocios, estructura de costos y logística que movilizan y son factores determinantes del comercio, por lo cual no debe concebirse como un procedimiento aislado, al contrario implica una compleja interacción de factores.

La magnitud y dimensión de la cooperación, significa la búsqueda del consenso interno tanto en Venezuela como en Nicaragua, para establecer cuáles son los sectores y bajo qué mecanismos y estrategias se inicia el proceso de cooperación, lo cual, significa el proceso de toma de decisiones con la concientización de ventajas y desventajas que se plantean en el contexto del comercio internacional.

Michael Porter, realizó un significativo aporte con la propuesta de la competitividad de las naciones; en el sentido que el hecho de hacer seguimiento y control de la producción, además de asumir la aptitud y disposición es lo que realmente permite a las empresas mantener los productos en el mercado, e incluso extender a otros Estados, la especialización de cada país en un área específica constituye un mecanismo para incentivar la ventaja comparativa y competitiva y dicha premisa necesariamente no tiene por qué ser una contradicción con el comercio internacional, al contrario contribuye al estímulo del mismo.

\section{Lista de referencias}

Calvo, A. (2016). Economía Internacional y Organismos Económicos Internacionales. Editorial Universitaria Ramón Areces Madrid. $2^{\text {a }}$ Edición

Churión J. (2001). Economía al Alcance de Todos. ALFA Grupo Editorial Caracas, Venezuela $4^{\mathrm{a}}$ edición

Hindle, T. (2008). Editorial El Comercio. Lima, Perú

León, F. (1991). Michael Porter, la Ventaja Competitiva de las Naciones. [Documento en Línea] (Disponible: eva.universidad.edu.uy)

Parkin. M. (2006). Microeconomía. Editorial Pearson Educación México. D.F. Séptima Edición. 
Pérez, R. (2004). El Desarrollo y la Opción Neoliberal (Reedición). Ediciones del Instituto Tecnológico de Santo Domingo, Santo Domingo

Ramírez, Z. (2006). Empresas Transnacionales e Integración en América Latina y el Caribe: La Grannacional, Una Alternativa Necesaria y Viable. (Tesis No Publicada) (Disponible: www.eumed.net/)

Rodríguez, S. (2010). Armonización Legislativa En Materia De Salvataje De Empresas En El Mercosur: ¿Una Quimera O Una Necesidad? [Versión Electrónica]. (Disponible: www.eumed.net/ ) 